\title{
Automatic Segmentation of the Ventricular System from MR Images of the Human Brain
}

\author{
H. G. Schnack, H. E. Hulshoff Pol, W. F. C. Baaré, M. A. Viergever,* and R. S. Kahn \\ Department of Psychiatry, A01.126, University Medical Center Utrecht, Heidel berglaan 100, 3584 CX Utrecht, The Netherlands; and \\ *Image Sciences Institute, University Medical Center Utrecht, Utrecht, The Netherlands
}

Received October 13, 2000; published online May 11, 2001

\begin{abstract}
An algorithm was developed that automatically segments the lateral and third ventricles from T1weighted 3-D-FFE MR images of the human brain. The algorithm is based upon region-growing and mathematical morphology operators and starts from a coarse binary total brain segmentation, which is obtained from the 3-D-FFE image. Anatomical knowledge of the ventricular system has been incorporated into the method in order to find all constituting parts of the system, even if they are disconnected, and to avoid inclusion of nonventricle cerebrospinal fluid (CSF) regions. A test of the method on a synthetic MR brain image produced a segmentation overlap of 0.98 between the simulated ventricles ("model") and those defined by the algorithm. Further tests were performed on a large data set of 227 1.5 T MR brain images. The algorithm yielded useful results for $98 \%$ of the images. The automatic segmentations had intraclass correlation coefficients of $\mathbf{0 . 9 9 6}$ for the lateral ventricles and 0.86 for the third ventricle, with manually edited segmentations. Comparison of ventricular volumes of schizophrenia patients compared with those of healthy control subjects showed results in agreement with the literature. 02001 Academic Press

Key Words: segmentation; ventricle; brain.
\end{abstract}

\section{INTRODUCTION}

Ventricular volume is a frequently examined feature of human brain development and abnormality. In a number of brain diseases it has been shown that the lateral and/or third ventricles are enlarged, e.g., in schizophrenia (for review see Wright et al., 2000), in depression (Ames and Chiu, 1997), and in dementia (Soares and Mann, 1997). MR imaging has made it possible to obtain in vivo three-dimensional (3-D) images of the human brain noninvasively, thereby allowing large scale examinations of the brains of patients and heal thy control subjects. While for some diagnostic applications a simple counting of the number of slices in which the ventricles appear, or even a visual inspec- tion, suffices, objective segmentation of the ventricular system is necessary for precise quantitative measurement of ventricular volume. Subtle differences in ventricular volume, as they appear in, e.g., schizophrenia (McCarley et al., 1999; Wrigth et al., 2000; Lawrie and Abukmeil, 1998), require an accurate segmentation algorithm. The relatively small deviations from normal ventricular volume variation and the large number of data sets in such studies, comparing patients to healthy control subjects, require an objective and reproducible segmentation method.

Segmentation of the ventricular system from MR images can be divided into two steps. F irst, cerebrospinal fluid (CSF) voxels have to be separated from brain tissue voxels, e.g., by means of thresholding on the voxel intensities. Second, the ventricular CSF voxels have to be separated from the other CSF voxels: cisterns and sulcal CSF. It is this second step that poses most problems in segmenting the ventricular system.

In a number of recently published studies (Rossi et al., 2000; Buchsbaum et al., 1997; Visser et al., 1999; Mu et al., 1999) the two steps are combined in a manual delineation procedure of (part of) the ventricular system. A frequently used solution of the segmentation problem is a two-step CSF/brain tissue separation, where thresholding or a more sophisticated (automated) algorithm is followed by interactive application of mathematical morphology operations to isolate the ventricles (Höhne and Hanson, 1992) or by manual outlining of the regions within which the ventricles' voxels are located (Woodruff et al., 1997; Blatter et al., 1997; Puri et al., 1999; Saeed et al., 1998; Leonard et al., 1999; Roy et al., 1998; Kitagaki et al., 1998; Coffey et al., 1998, 1999; Worth et al., 1998). In high resolution images, with a large number of slices, this is tedious work, especially if one wishes to include all, often apparently disconnected, parts of the system. Moreover, the separation from the ventricles of cisterns and sulci near the extreme parts of the ventricular system is a delicate operation, as boundaries are often not clear. In these segmentation procedures, and even more so in the fully manual delineations, the 
results depend strongly on intra- and interrater variability.

The subjectivity and irreproducibility of (partly) manual segmentations have stimulated the search for automated-or at least objective and reproducible segmentation algorithms. Mclnerney and Terzopoulos (1999) applied T-snakes, which can modify their topology if necessary. Declerck et al. (1995) used a nonlinear warping technique to segment the ventricles, based on a segmented model brain image. However, the wide range of ventricular shapes with apparently disconnected parts embedded within sulcal offshoots and cisterns seem to hinder a straightforward segmentation in both approaches. Hojjatoleslami et al. (1999) separated CSF from tissue by an automatic method, after which they apply cutting planes to bound the ventricular CSF regions. This is a rather coarse way of segmenting the ventricles and it does not discriminate between lateral and third ventricles.

In this work, we present a method that, after an automatic CSF/brain tissue separation within the intracranial volume, automatically classifies the lateral and third ventricular CSF voxels in a brain image. The algorithm is designed to include all ventricle parts, even if they appear disconnected on the image, and a discrimination between lateral and third ventricles is made; for the third ventricle only, a few artificial boundaries are necessary. The algorithm is based on region-growing and mathematical morphology operations and anatomical knowledge. A first evaluation of the algorithm was done on a simulated MR brain image of which the underlying segmentation is known. The algorithm was then applied to two sets (227 and 20 images, respectively) of real MR data.

\section{MATERIALS AND METHODS}

A 3-D-FFE T1-weighted image (TE = $4.6 \mathrm{~ms}$; TR = $30 \mathrm{~ms}$; flip angle $=30^{\circ}$ ), with voxel dimensions $1 \times 1 \times$ $1.2 \mathrm{~mm}^{3}$ is positioned in Talairach orientation (no scaling) (Talairach and Tournoux, 1988), with voxel length $1.2 \mathrm{~mm}$ in the anterior-posterior direction. A coordinate system (xyz) in millimeters is defined for the image. In this system, $x$ runs from subject's right to left, $z$ runs from superior to inferior, and y runs from anterior to posterior. The xz plane is the coronal plane, the $y z$ plane is the sagittal plane, and the xy plane is the transversal, or axial, plane. A (coarse) total brain (i.e., gray plus white matter tissue) and a (coarse) white matter segmentation (a simple thresholding will suffice; see also, e.g., Schnack et al., 2001) are segmented from the image. The size of the brain is defined by the distances between the extreme points of the total brain segment, in the three directions, which are denoted by $L_{x}, L_{y}, L_{z}$. Parts of the segmentation algorithm involve search areas, the sizes of which will be defined relative to these measures. For this, "standard" brain sizes are given by $\mathrm{L}_{\mathrm{x}_{\text {sts }}}=132 \mathrm{~mm}, \mathrm{~L}_{\mathrm{y}_{\text {std }}}=174$ $\mathrm{mm}, \mathrm{L}_{z_{\text {std }}}=145 \mathrm{~mm}$. These "standard" sizes are averages over 227 test brains (see Testing and Evaluation). For segmentation of the third ventricle, the coordinates of the anterior commissure (AC) and posterior commissure (PC) are needed, as well as a bounding plane for the third ventricle (this is necessary because the bounding membranes of the third ventricle are not well visible on MR images, due to the partial volume effect).

The total brain segmentation is a binary image, consisting of voxels with value 1 at places with brain tissue and voxels with value 0 at other places: cerebrospinal fluid (CSF) and all other voxels surrounding the brain. The remaining and most complex part of the problem is the classification of the correct zero-valued voxels in the total brain image as ventricular CSF.

The ventricle segmentation algorithm is based on the following anatomical principles: (1) Ventricles are encapsulated by white matter. (2) The ventricular system is a connected structure and could, therefore, in principle, be segmented with one region-growing operation. However, owing to the partial volume effect, parts of the system appear not connected on the image, and additional anatomical knowledge of the shape of the system needs to be inserted in the algorithm to find these disconnected parts. The same applies to the removal of apparently connected nonventricular CSF regions (cisterns, sulci). In practice, this means that the region-growing is split up in several steps and that per growing step abnormal extensions are detected and fixed. To facilitate this, we define subregions of the ventricular system, which can be segmented separately, if they appear disconnected from each other. Knowledge of the anatomy of the ventricular system is inserted to guide the algorithm in its search for subregions. This information is in the form of relative positions and sizes of subregions with respect to each other, as will be discussed in the following sections. The subregions include the left $(L)$ and right $(R)$ main bodies of the lateral ventricles, $L$ and $R$ anterior tips of the lateral ventricles, $\mathrm{L}$ and $\mathrm{R}$ posterior tips of the lateral ventricles, $L$ and $R$ temporal horns of the lateral ventricles, and the third ventricle. Figures $1 a$ and b provide schematic views of the different parts of the ventricular system. The block diagram (Fig. 1c) shows the consecutive segmentation steps of the algorithm. In the following the segmentation procedure is described for each ventricular subregion.

\section{Segmentation of the Lateral Ventricles}

Segmentation is done in axial slices, globally in superior-to-inferior direction.

1.1. The upper parts $(M)$ of the lateral ventricles. Slice by slice, starting from the top of the image downward, the first appearance of an island of zeros en- 


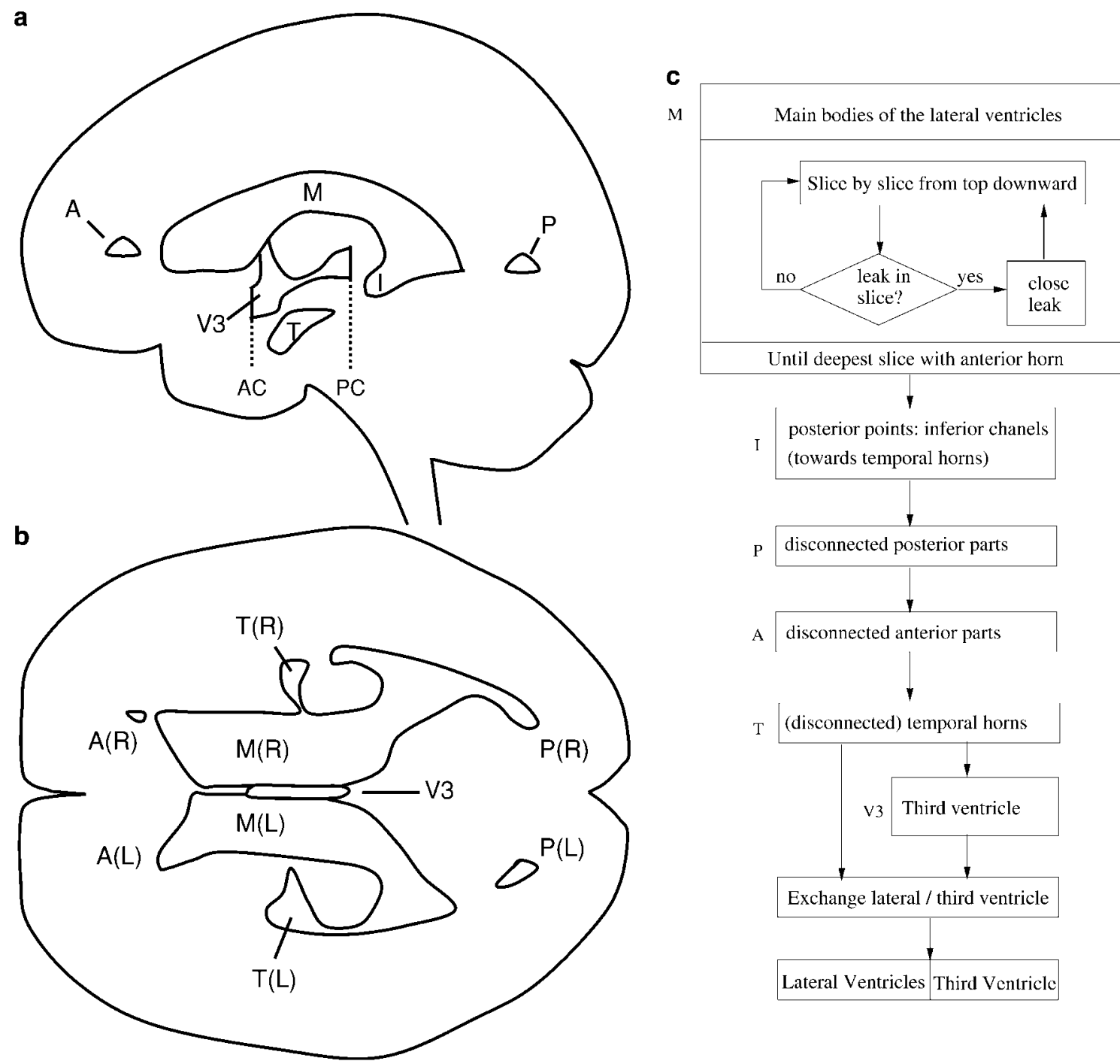

FIG. 1. Schematic views of the ventricular system in (a) sagittal projection and (b) axial projection. The following subregions are defined for segmentation purposes: the main bodies of the lateral ventricles (M); the inferior parts of the main bodies (I); the temporal horns ( $T$ ); the anterior tips (A); the posterior tips (P); the third ventricle (V3), limited by the AC plane and the PC plane. In the axial projection (b), one can discriminate between the left $(\mathrm{L})$ and right $(\mathrm{R})$ subregions. (c) Block diagram, which shows the consecutive segmentation steps for the subregions of (a) and (b).

closed by white matter is searched for. This is done for $L$ and $R$ separately. The points which are found are the starting points of two region-growing operations. Figures $2 a$ and $b$ show the first axial slice in which both islands are present. We use a unidirectional downward (increasing z) 3-D region-growing operator, which prevents possible leaks to cisterns and sulci from migrating upward. Slice by slice the grown areas are examined upon leaking. Each time the region-growing operation of a slice with axial coordinate $z$ has finished, the grown area in this slice is compared with that of the previous slice (z-1). This is done by projecting these two slices on top of each other in the $(x, y)$ plane. In this projection plane a search is done for voxels grown in the lower slice ( $z$ ) but not grown in the previous slice (z-1). These are normally due to the downward broadening or, more generally, change of shape. However, a sudden appearance of a large connected area of such voxels is possibly a leak into a cistern. If such a region crosses the midsagittal plane of the brain, this region is considered to be cistern. The zero-valued voxels of this region in the total brain segmentation are set to a value different from zero and the last region-growing step is carried out again. This time the "wrong" region cannot be grown. This procedure is repeated until all growing is done. The lateral ventricle growing stops when there are no more connected voxels left to add to the segmentation. This segmentation is called S1 and includes at least the main bodies (M) of the lateral ventricles. Starting from segmentation S1, in four 
a

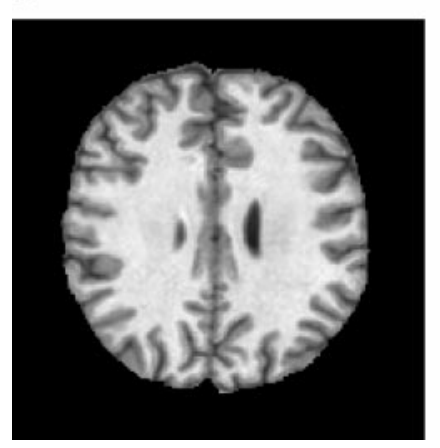

b

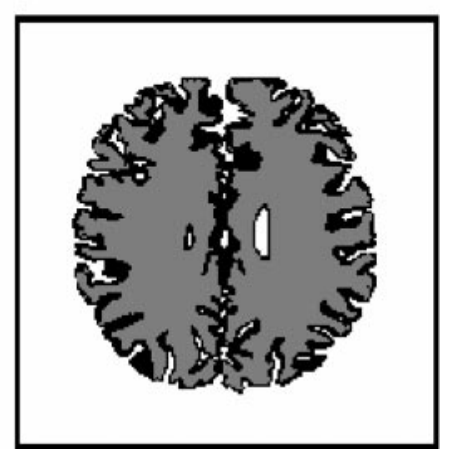

FIG. 2. (a) Axial slice of a brain image at the level where the main bodies of the lateral ventricles appear. (b) Segmentation of the slice of (a). The following gray tones were used: white, CSF and "outside" (including nonbrain tissue); gray, "overestimated white matter" (the overestimation was on purpose); black, other brain tissue ("gray matter").

steps (1.2-1.5) additional parts are grown, if they have not yet been included in S1.

1.2. The inferior parts (I) of the main bodies of the lateral ventricles. In the most inferior slice of segment S1 the most posterior points of S1 are searched for, $L$ and $R$. These points serve as starting points for $a$ further downward unidirectional 3-D region-growing of the lateral ventricles (I). These growings will either end up in the temporal horns (T), or stop earlier, because the connections are too thin to be visible on the MRI, with the current resolution (the latter turns out to happen in most cases). Possible leaks to the "outside" are detected by a sudden step in the volume that is grown per axial slice $(L, R)$. All growing from this slice downward is canceled. The obtained segment is called S2, and it consists of M plus I, and possibly $\mathrm{T}$.

1.3. The "disconnected" posterior tips $(P)$ of thelateral ventricles. In S2, the most posterior points are searched for, $L$ and $R$ (see Fig. 3). We denote the coordinates of such a point by $\left(x_{p p}, y_{p p}, z_{p p}\right)$. For $L$ and $R$, a box is defined with one corner at $\left(x_{p p}, y_{p p}, z_{p p}\right)$ and the diametral corner at $\left(\mathrm{x}_{\mathrm{pp}} \pm \mathrm{dx} \mathrm{x}_{\mathrm{pp}}, \mathrm{y}_{\mathrm{pp}}+\mathrm{dy}_{\mathrm{pp}}, \mathrm{z}_{\mathrm{pp}}-\mathrm{d} \mathrm{z}_{\mathrm{pp}}\right)$, where the plus-sign is for $R$ and the minus-sign for $L$. The dimensions of the box depend on the size of the brain (see Appendix A for details). In this box zerovalued voxels are searched for. These points serve as seed points for 3-D region-growing operations. If the resulting regions are bound, they are classified as "disconnected" parts of the posterior lateral ventricles (P).

1.4. The "disconnected" anterior tips $(A)$ of the lateral ventricles. A procedure comparable to the one de scribed for the posterior tips (Subsection 1.3) is carried out for the anterior tips. In S2, the most anterior points are searched for, $L$ and $R$. We denote the coordinates of such a point by $\left(x_{a a}, y_{a a}, z_{a a}\right)$. For $L$ and $R$, a box is defined with one corner at $\left(\mathrm{x}_{\mathrm{aa}}, \mathrm{y}_{\mathrm{aa}}, \mathrm{z}_{\mathrm{aa}}\right)$ and the diametral corner at $\left(\mathrm{x}_{\mathrm{aa}} \pm \mathrm{dx}_{\mathrm{aa}}, \mathrm{y}_{\mathrm{aa}}-\mathrm{dy}_{\mathrm{aa}}, \mathrm{z}_{\mathrm{aa}}-\mathrm{d} \mathrm{z}_{\mathrm{aa}}\right)$, where the plus-sign is for $L$ and the minus-sign for $R$. The dimensions of the box depend on the size of the brain (see Appendix A for details). In this box zero-valued voxels are searched for. These points serve as seed points for 3-D region-growing operations. If the resulting regions are bound, they are classified as "disconnected" parts of the anterior lateral ventricles (A). The union of segments S2, A and P is called S4.

1.5. The temporal horns $(T)$ of the lateral ventricles. The temporal horns of the lateral ventricles are segmented only if they are not connected to the main body in segment S4. The principle of finding them similar to that used for the main bodies of the lateral ventricles. Starting from the most inferior slice of the image in which the total brain segment is visible, in each slice $L$ and $\mathrm{R}$ isolated regions of zero-valued voxels are searched for, which are connected to the white matter temporal lobes. However, there are many different regions in this part of the brain that look like ventricle parts (see Fig. 4). Therefore, a number of criteria concerning size, shape, and orientation is applied to the found regions: These are described in A ppendix A. CSF regions satisfying all criteria are further grown with unidirectional upward (decreasing z) 3-D region-growings. The region-growing operations are necessary, because in some slices the criteria may be too strict to accept a proper CSF region as ventricle. A regiongrowing operation that leads to a leak to the "outside" (detected by a sudden step in the volume that is grown per axial slice) is canceled from the slice where the leaking starts upward. After all region-growings have been carried out, the resulting segments are merged with segment S4, yielding segment S5.

\section{Segmentation of the Third Ventricle}

For the segmentation of the third ventricle (V3) some artificial boundaries are used. This is a necessary step

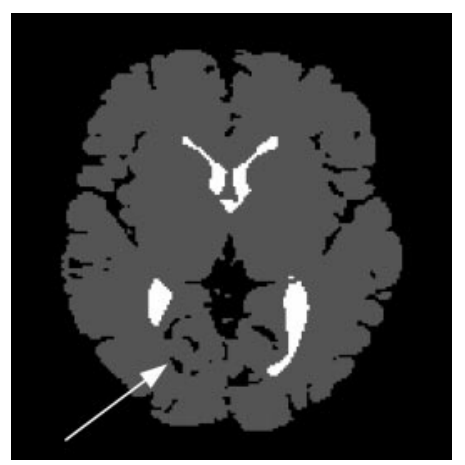

FIG. 3. Axial slice of the brain image of Fig. 2, $26 \mathrm{~mm}$ inferior to the slice of Fig. 2. The total brain segment is shown in gray; the ventricles grown so far in white. Other CSF and "outside" are shown in black. The arrow indicates a posterior part of the right lateral ventricle that is apparently not connected to the main body on the image, because the connection is too thin. The algorithm (Materials and Methods, step 1.3) automatically finds and grows this piece and discards the other nearby loose ends of sulci and cisterns. 


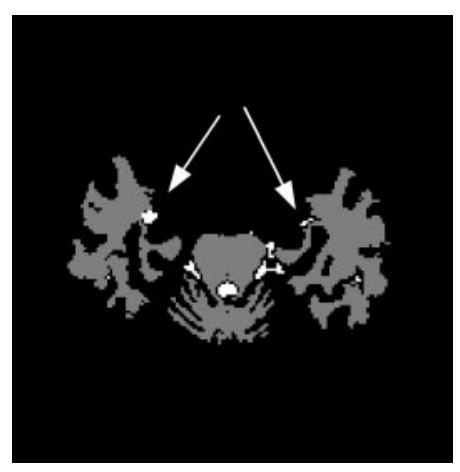

FIG. 4. Axial slice of a brain image through the temporal lobes. The following gray tones were used: black, "outside"; gray, "overestimated white matter"; and white, isolated CSF spots connected to the white matter lobes. The two spots indicated with arrows meet the criteria for being temporal horn (Materials and Methods, step 1.5).

because its bounding membranes, which separate it from the surrounding cisterns, are so thin that the partial volume effect makes them invisible in most images. For lower resolution images, this effect will be even more pronounced. The anterior and posterior boundaries are given by the coronal (zx) planes through the anterior commissure (AC) and posterior commissure (PC), respectively. The superior boundary is given by a plane through the plexus choroideus and ventriculi tertii in the midsagittal slice perpendicular to this slice (parallel to the $x$-axis). With these boundaries defined, a seed point is automatically searched for between the AC and PC planes, in, or close to, the midsagittal plane. A simple region-growing suffices to segment the third ventricle. To avoid leaking of this operation to the "outside," it is performed on the "closed" brain segmentation image, in order to avoid leaks to the outside. First a closing (dilation followed by erosion (Serra, 1982)) of the total brain segment is performed. In the resulting image, a region-growing of the "outside" is done, which segment, when put in the original total brain segmentation image with a value different from 1, serves as a "stop" for the regiongrowing of the third ventricle. The third ventricle segment is called S3.

\section{IMPLEMENTATION}

The ventricle segmentation algorithm is implemented in a (UNIX) shell script calling compiled C and $\mathrm{C}++$ programs. The script consists of a large series of region-growing and mathematical morphology operations, image search algorithms and histogram analysis algorithms. The run time of the segmentation algorithm varies from about 5 to 20 min on a Pentium3 450 $\mathrm{MHz} \mathrm{PC}$, depending on the complexity of the ventricular system of the image and the severeness of the leaks. The modular form of the program is especially important in the development phase, because of its flexibility and because of the logging of actions and problems. For routine use, however, the run time of the algorithm may be reduced significantly (to about 1 min) by converting it to a single $\mathrm{C}$ program.

\section{TESTING AND EVALUATION}

\section{Ventricle Segmentation}

The automatic ventricle segmentation algorithm is first tested on a single 3-D-FFE image (TE = 4.6 ms; $\mathrm{TR}=30 \mathrm{~ms}$; flip angle $=30^{\circ}$ ), of which the results are shown in Fig. 5 in a number of axial slices. A threedimensional rendering of the segmentation is shown in Fig. 6. The "raw" automatic segmentation needs to be checked visually, and edited if necessary. Most frequent problems are parts of the temporal horns which could not be segmented (they were either missed or could not be grown due to severe leakages) and third/ lateral ventricle distinction errors in cases where there is no clearly visible border between them. The feedback from logfile and editing is used continuously to improve the segmentation algorithm further.

\section{Evaluation on the BrainWeb (MNI)-Simulated Brain MR Scan}

A synthetic T1-weighted MR image was obtained from the BrainWeb (Cosoco et al., 1997; Kwan et al., 1996; Collins et al., 1998). Voxel size was $1 \times 1 \times 1$ $\mathrm{mm}^{3}$. Noise percentage was $3 \%$. Of this image a total brain segmentation and white matter segmentation was made, following our standard image processing procedure, as described in the following section. The automatic ventricle segmentation program was run with these images and AC, PC, and roof coordinates information as inputs.

A "model ventricle segment" was created in the following way. A segmentation in ten (tissue) classes was provided by BrainWeb. An experienced rater edited the CSF segment, keeping only the ventricles. For the third ventricle, the same rules (AC, PC, roof) as for the automatic procedure were applied. A "model total brain" was created as well, by selecting the model's gray matter, white matter, and "glial matter" voxels. Now the automatic ventricle segmentation program was run again, with our total brain segmentation replaced by this "model total brain." By doing this, we removed the effect of CSF/brain tissue thresholding, so that we could investigate the capability of the al gorithm to collect the right CSF-spaces as being ventride.

For comparison of the segmentation results, the overlap metric (see, e.g., Zijdenbos et al. (1994)) was calculated: $\varepsilon=2 \mathrm{~V}_{12} /\left(\mathrm{V}_{1}+\mathrm{V}_{2}\right)$, where $\mathrm{V}_{1}$ and $\mathrm{V}_{2}$ are the volumes of the two segments that are compared, and $\mathrm{V}_{12}$ is the volume of the overlap of the two segments. 

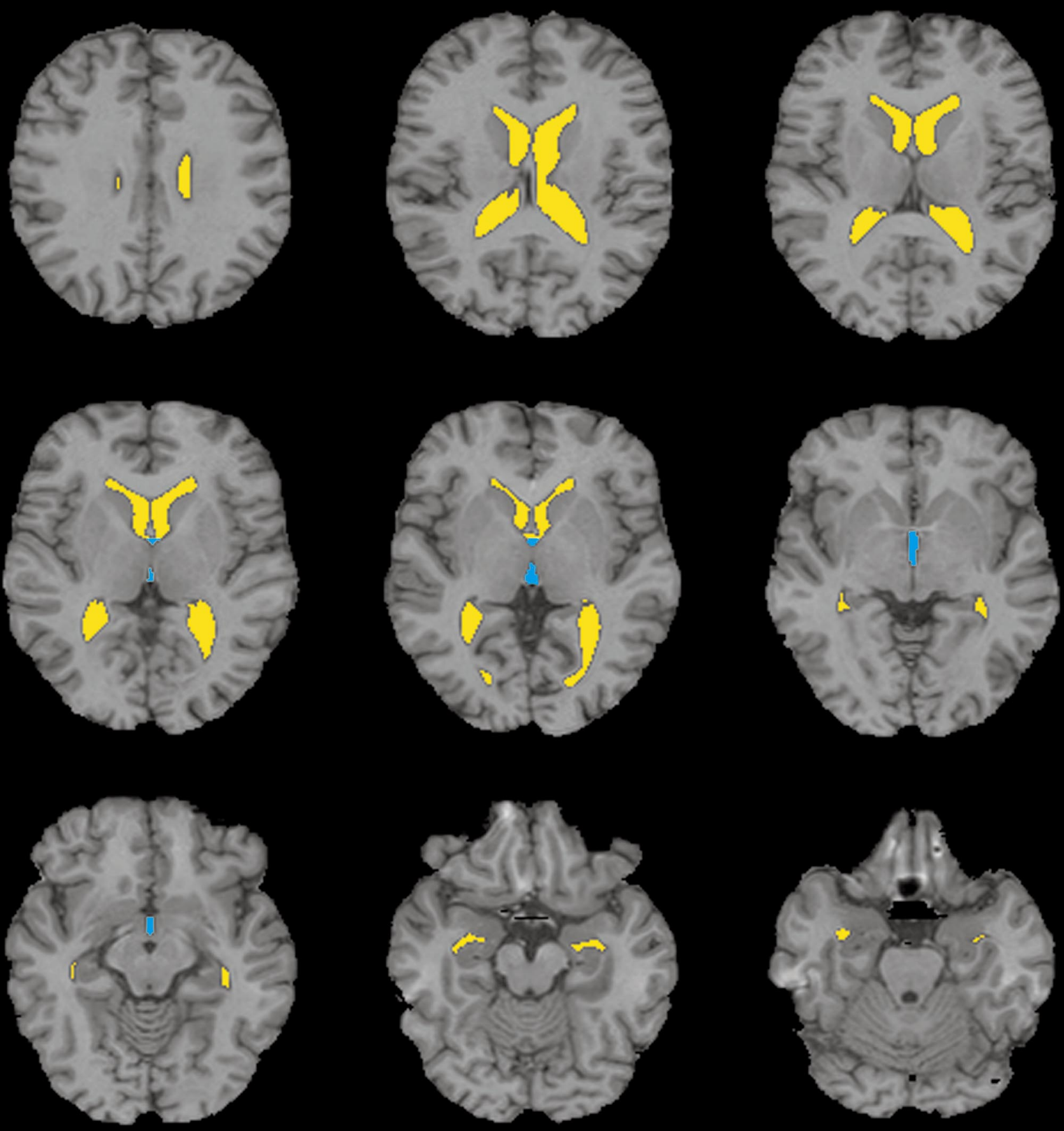

FIG. 5. Nine slices showing the ventricle segmentation by the automatic method. The slices are at $46,58,63,68,72,78,84,89,94 \mathrm{~mm}$ from the top of the brain. The lateral ventricles are shown in yellow; the third ventricle is shown in cyan.

The volume ratio of the computed and model ventricular volumes were calculated as well. The results are shown in Table 1. A large part of the voxels missed by the "raw" automatic segment VA1 is ventricle boundary, due to the $1 \%$ larger automatic total brain segmentation, as compared to the model total brain. This is an effect of the total brain segmentation procedure and not of the ventricle segmentation algorithm. It disappeared in VA2, where the "model total brain" was used.

\section{Evaluation on a Large Set of T1-Weighted I mages}

MRI brain scans from 227 subjects were randomly selected from the Schizophrenia Project database of the 


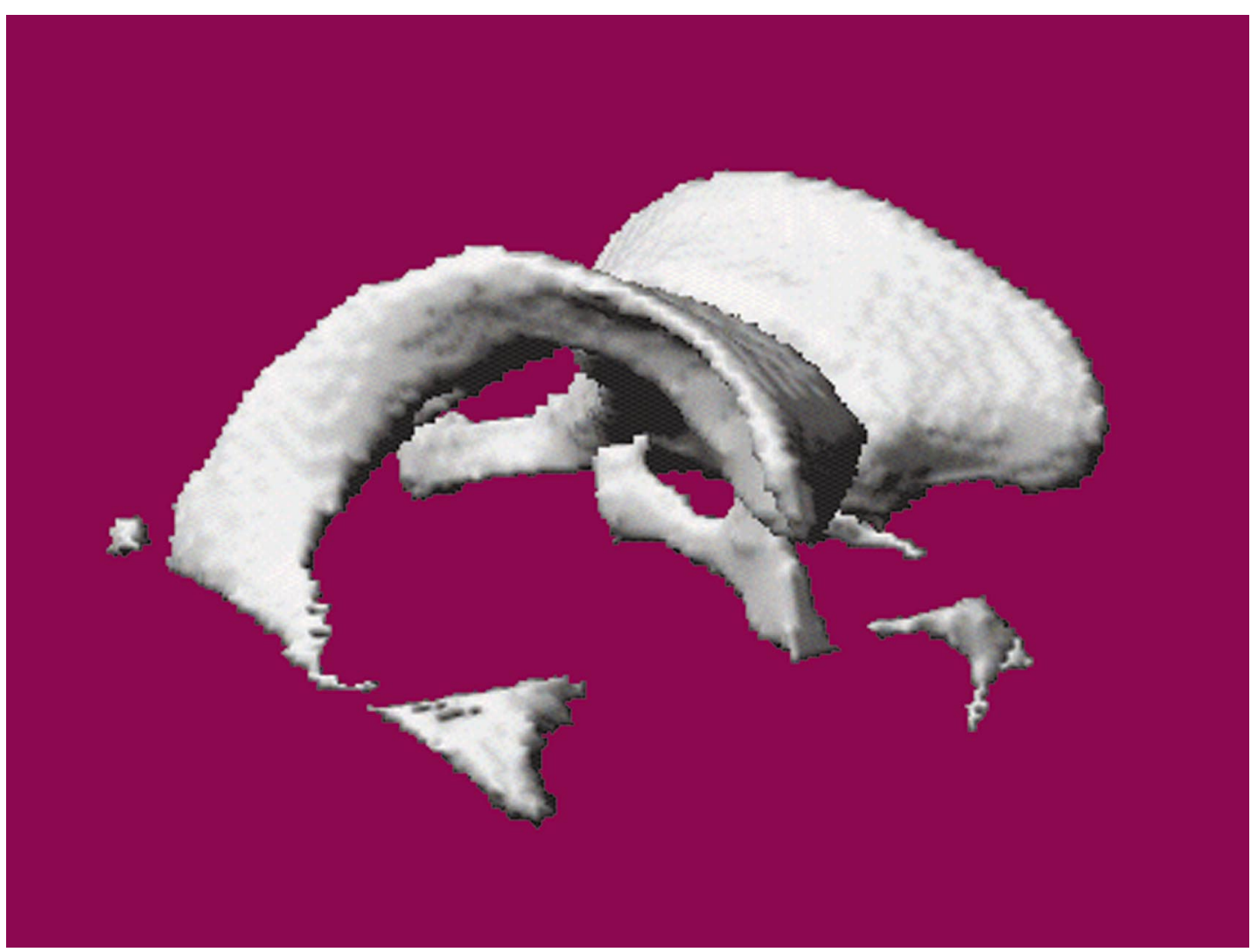

FIG. 6. Three-dimensional rendering of an automatically segmented ventricular system. Clearly visible are the left and right lateral ventricles, with apparently disconnected anterior and posterior tips. In the center of the ventricular system the third ventricle is shown.

Department of Psychiatry (Baaré et al., 2001; Cahn et al., 1999; Staal et al., 2000; Hulshoff Pol et al., 2000a). They included healthy controls, patients with a diagnosis of schizophrenia, and family members of patients with schizophrenia. They had participated in studies of the Schizophrenia Project after written informed consent was obtained.

The Magnetic Resonance images of the 227 subjects were obtained on a Philips 1.5 T scanner. T1-weighted scans with 160-180 1.2-mm contiguous coronal slices (TE $=4.6 \mathrm{~ms} ; \mathrm{TR}=30 \mathrm{~ms}$; flip angle $=30^{\circ} ; \mathrm{FOV}=256$ $\mathrm{mm} / 80 \% ; 1 \times 1 \mathrm{~mm}^{2}$ ) and dual contrast turbo spin echo (DTSE) scans with $1201.6 \mathrm{~mm}$ contiguous coronal slices (TE1 = $14 \mathrm{~ms}$; TE2 = $80 \mathrm{~ms}$; TR = $6350 \mathrm{~ms}$; $\mathrm{FOV}=256 \mathrm{~mm} / 80 \%$; $1 \times 1 \mathrm{~mm}^{2}$ ) of the whole head were acquired. After transforming the T1-weighted images into Talairach frame (no scaling) (Talairach and Tournoux, 1988), the DTSE images and the intracranial region segmentations on them were registered to the T1-weighted images by employing maximization of mutual information (Maes et al., 1997). Furthermore, the images were corrected for scanner RF field nonuniformity (Sled et al., 1998). This is a necessary step for segmenting the whole brain, which influences the (ventricular) CSF/gray matter border. The intensities of voxels belonging to the intracranial volume were di- vided by the so-called gain field, which describes the variations in RF field strength, calculated from the image. On the nonuniformity corrected T1-weighted image a total brain segmentation was made automatically. We used a series of mathematical morphology operators based upon intensity histogram analysis of the image (Schnack et al., 2001). A coarse white matter segmentation was obtained automatically by thresholding the T1-image with an intensity value roughly between the gray and white matter peaks in the histogram. A precise segmentation was not necessary as the white matter segment only serves as guide for the ventricle segmentation algorithm. The total brain and white matter segmentations were the inputs of the ventricle segmentation algorithm.

\section{TABLE 1}

Comparison of Automatic Segmentation Results on the BrainWeb Simulated I mage with the Model Segmentation

\begin{tabular}{lcc}
\hline \multicolumn{1}{c}{ Automatic run } & $\begin{array}{r}\text { Volume ratio } \\
\text { autom./model }\end{array}$ & Overlap metric \\
\hline VA1, run on our TB & 0.966 & 0.978 \\
VA2, run on model TB & 0.995 & 0.990 \\
\hline
\end{tabular}




\section{TABLE 2}

Influence of Editing the Automatic Ventricle Segmentations

\begin{tabular}{ccccc}
\hline & & \multicolumn{2}{c}{ Volume mean (SD) $\left(\mathrm{mm}^{3}\right)$} & \\
\cline { 2 - 4 } \multicolumn{2}{c}{ Segment } & Patient & Control & P value \\
\hline \multirow{2}{*}{ Lateral "Raw" } & $20087(12395)$ & $14996(8665)$ & 0.0063 \\
& Edited & $19760(11851)$ & $14716(7875)$ & 0.0039 \\
& Ratio & $1.0037(0.0074)$ & $1.0083(0.0043)$ & 0.6 \\
Third & "Raw" & $895(424)$ & $709(338)$ & 0.0061 \\
& Edited & $1037(505)$ & $823(443)$ & 0.0108 \\
& Ratio & $0.912(0.045)$ & $0.884(0.027)$ & 0.6 \\
\hline
\end{tabular}

This set of 227 images was used to check the results of automatically segmented ventricle volumes versus manually edited (starting from the automatic segmentations) volumes.

In all cases the algorithm succeeded in finding and segmenting the lateral ventricles. In five cases (2\%) it failed to find the third ventricle. We calculated overlap metrics $\varepsilon$ between "raw" automatic and edited segments. The mean (SD) overlap metric for the lateral ventricles (on 227 images) was $\varepsilon=0.989$ (0.018), and for the third ventricle (on 222 images) $\varepsilon=0.925$ (0.145). We also calculated the intraclass correlation coefficients (icc, see, e.g., Bartko and Carpenter, 1976) of the volume values of the segments between "raw" and edited segments. We found values of icc $=0.996$ for the lateral ventricles, and icc $=0.86$ for the third ventricle. In order to investigate the discriminative power of the algorithm when segmenting the ventricles of schizophrenia patients and healthy control subjects, 74 patients and 56 healthy control subjects (no family members of patients) from the set, of which both the "raw" and "clean" segmentations were available, were compared. Average ventricular volumes for the two groups were calculated, both "raw" and edited. The results are shown in Table 2 . From the table it can be seen that the mean lateral ventricle volumes differ only marginally. For the third ventricle, the difference is larger, but has about the same size and sign for the two groups. When comparing the mean volumes of lateral and third ventricles between patients and healthy control subjects, it is seen that the significance of the differences is not affected by editing. The volume ratios "raw"/edited did not differ significantly between patients and healthy control subjects, showing that there was no bias between the groups.

The MR brain scans of some of these subjects were included in a large study (Hulshoff Pol et al., 2000b), where ventricle volume ratios of patients with schizophrenia as compared to healthy comparison subjects of 1.30 (lateral) and 1.27 (third) were found, in agreement with values found in a recent meta analysis of volumetric studies in schizophrenia patients and control subjects (Wright et al., 2000).

\section{Application to I mages of Megalencephaly Patients}

The capability of the algorithm to segment the ventricular system on images that are much different from the images of the large data set, for which the algorithm was developed, was tested in a study on megalenchepaly. This set included eight megalencephaly patients (age range 5-21 year) and 12 age-matched healthy control subjects (Braun et al., 2000). The brains of children are smaller and of different shape than adult brains, and the brains of the megalencephaly patients deviate even more, in size, shape, and relative constitution of brain and CSF. Furthermore, the voxel size of these scans was $1 \times 1 \times 3.6 \mathrm{~mm}^{3}$, which is of much lower resolution than the $1 \times 1 \times 1.2$ $\mathrm{mm}^{3}$ images used for development. On one of the 20 images the algorithm failed to run correctly; the third ventricle could not be found on 5 of the remaining 19 images. The 19 ventricle segmentations were checked and edited if necessary. I ntraclass correlation coefficients were calculated between "raw" and edited segments. For the lateral ventricles we found icc $=0.997$ (on 19 images); for the third ventricle we found icc = 0.791 (on 14 images).

\section{DISCUSSION}

In this work we developed an automated ventricle segmentation method, designed for application to 3-DFFE high resolution MR human brain images. However, application to various other types of MR human brain images should be possible, as no explicit use of the scan type is made. The method segments the lateral ventricles and the third ventricle. The ventricular system is segmented by region growing and mathematical morphology operations, applied to the binary total brain image which is obtained from the original image by presegmentation. The region growing is guided by a coarse white matter segmentation and by a priori defined anatomical knowledge of the ventricular system to determine which CSF regions are ventricle and which are not. The algorithm was tested on a BrainWeb synthetic image and on a large set of 3-D-FFE images from the Utrecht neuroimaging database. A test on a set of megalencephaly patients was carried out to test the algorithm on images of brains which differ considerably from the adult brain images of the other test sets. Volumetric results were compared with values found in the literature.

These tests showed that the algorithm gives qualitatively (visual check of the segmentations) and quantitatively (comparison with values from the literature) valid ventricle segmentations in $98 \%$ of the high resolution images, where the few non-valid results were easily detected and repaired. The lateral ventricles were always found and the segmentations showed excellent agreement (icc $=0.996$ ) with the manually ed- 
ited segments. The third ventricle was found in $98 \%$ of the cases. When compared to manually edited segmentations, an icc of 0.86 was found. This value is relatively low compared to the very high value for the lateral ventricles. On the one hand, this is due to the small dimensions of the third ventricle. On the other hand, the third ventricle region suffers from many leaks which often prevent the algorithm to include all slices of the third ventricle.

The test on the megalencephaly set showed that the algorithm performs well on aberrant brain images as well: for the lateral ventricles an icc of 0.997 (on 19 images) was found. The third ventricle segmentation part of the algorithm has to be improved for this kind of images, as in five of the 19 images it failed to give proper third ventricle segmentations. For the remaining 14 images an icc of 0.791 was found.

For more precise measurements of (parts of) the ventricular volumes, the automatic segmentation can be edited, although it was shown that the editing did not alter the level of significance of the difference in ventricular volume found between schizophrenia patients and healthy controls. The feedback from the editing can be used to further improve the algorithm. The modular design of the algorithm facilitates modification of parts of the segmentation procedure, without altering the well functioning modules.

In conclusion, we have developed an automated ventricle segmentation algorithm, which turned out to give reliable results.

\section{APPENDIX A}

In this appendix information is provided about some details of the segmentation algorithm. The values of constants were found empirically during the developing phase of the algorithm. The sensitivity of the algorithm for these constants is low, as they are mainly involved in definitions of search areas.

Step 1.3. The dimensions of the box depend on the size of the brain and are given by: $d x_{p p}=0.046 \times L_{x}$, $\mathrm{dy}_{\mathrm{pp}}=0.121 \times \mathrm{L}_{\mathrm{y}}, \mathrm{dz} \mathrm{z}_{\mathrm{pp}}=0.069 \times \mathrm{L}_{\mathrm{z}}$. These measures are in $\mathrm{mm}$; in the algorithm as implemented in a computer program, they are converted to dimensionless coordinates, by dividing them by the voxel lengths.

Step 1.4. The lengths of the edges of the box are given by: $\mathrm{dx}_{\mathrm{aa}}=0.068 \times \mathrm{L}_{\mathrm{x}}, \mathrm{dy}_{\mathrm{aa}}=0.055 \times \mathrm{L}_{\mathrm{y}}, \mathrm{dz}_{\mathrm{aa}}=$ $0.021 \times \mathrm{L}_{z}$.

Step 1.5. The following criteria are used: (1) The area of a CSF region has to be at least $3.6 \mathrm{~mm}^{2} \times\left(\mathrm{L}_{x}\right.$ $\left.\times L_{y}\right) /\left(L_{x_{-} \text {std }} \times L_{y_{-} s t d}\right)$; (2) The center of mass (COM) of a CSF region has to have a distance to the midsagittal plane of at least $20 \mathrm{~mm} \times\left(\mathrm{L}_{x} / \mathrm{L}_{\mathrm{x}_{-} \text {std }}\right)$; (3) The COM of the WM lobe to which the CSF region is attached is also calculated. The angle $\vartheta$ of the line segment COM(WM
lobe)-COM(CSF region), relative to the x-axis, is limited in the following way: $-28.5^{\circ} \leq \vartheta \leq 44.5^{\circ}$.

\section{ACKNOWLEDG MENTS}

We thank M. L. C. Hoogendoorn and W. G. Staal for their assistance with the data processing. We also thank K. P. J . Braun for the data of the megalencephaly set. Furthermore, for software, we are grateful to the Department of Biomedical Engineering, McGill University, Montreal, and the Laboratory for Medical I maging Research, K.U. Leuven.

\section{REFERENCES}

Ames, D., and Chin, E. (Eds.) 1997. Neuroimaging and the Psychiatry of Late Life Cambridge Univ. Press.

Baaré, W. F. C., Van Oel, C. J ., Hulshoff Pol, H. E., Schnack, H. G., Durston, S., Sitskoorn, M. M., and Kahn, R. S. 2001. Volumes of brain structures in twins discordant for schizophrenia. Arch. Gen. Psychiatry 58: 33- 40.

Bartko, J . J ., and Carpenter, W. T. 1976. On the methods and theory of reliability. J . Nervous Mental Dis. 163: 307-317.

Blatter, D. D., Bigler, E. D., Gale, S. D., J ohnson, S. C., Anderson, C. V., Burnett, B. M., Ryser, D., Macnamara, S. E., and Bailey, B. J . 1997. MR-based brain and cerebrospinal fluid measurement after traumatic brain injury: Correlation with neuropsychological outcome. Am. J . Neuroradiol. 18: 1-10.

Braun, K. P. J ., Hoogendoorn, M. L. C., Hulshoff Pol, H. E., Schnack, H. G., Witkamp, T. D., Gooskens, R. H. J . M., and Van der Grond, J. 2000. ${ }^{1} \mathrm{H}$ MR imaging and volumetry in idiopathic anatomic megalencephaly. Proc. Intl. Soc. Magn. Res. Med. 8: 1934.

Buchsbaum, M. S., Yang, S., Hazlett, E., Siegel, B. J ., Germans, M., Haznedar, M., O'Flaithbheartaigh, S., Wei, T, Silverman, J ., and Siever, L. J . 1997. Ventricular volume asymmetry in schizotypal personality disorder and schizophrenia assessed with magnetic resonance imaging. Schizophrenia Res. 27: 45-53.

Cahn, W., Hulshoff Pol, H. E., Bongers, M., Lemstra, A., Schnack, H. G., Van Haren, N., Van der Weele, W., Van der Schot, A., Van der Linden, J. A., and Kahn, R. S. 1999. Brain morphology in antipsychotic-naive, first-episode schizophrenia. Schizophrenia Res. 36: 192. [Abstract]

Coffey, C. E., Lucke, J . F., Saxton, J . A., Ratcliff, G., Unitas, L. J ., Billig, B., and Bryan, R. N. 1998. Sex differences in brain aging. Arch. Neurol. 55: 169-179.

Coffey, C. E., Saxton, J . A., Ratcliff, G., Bryan, R. N., and Lucke, J . F. 1999. Relation of education to brain size in normal aging. Implications for the reserve hypothesis. Neurology 53: 189-196.

Collins, D. L., Zijdenbos, A. P., Kollokian, V., Sled, J. G., Kabani, N. J ., Holmes, C. J ., and Evans, A. C. 1998. Design and construction of a realistic digital brain phantom. IEEE Trans. Med. Imaging 17: 463- 468.

Cosoco, C. A., Kollokian, V., Kwan, R. K.-S., and Evans, A. C. 1997. BrainWeb: Online interface of a 3-D MRI simulated brain database. Neurol mage 5 (4) part 2/4: S425. (http://www.bic.mcgill.ca/ brainweb/)

Declerck, J., Subsol, G., Thirion, J.-P., and Ayache, N. 1995. Automated retrieval of anatomical structures in 3D medical images. In Lecture Notes in Computer Science (N. Ayache, Ed.), Vol. 905, pp. 153-162. Springer.

Höhne, K. H., and Hanson, W. A. 1992. Interactive 3-D segmentation of MRI and CT volumes using morphological operations. J . Comput. Assist. Tomogr. 16: 285-294.

Hojjatoleslami, S. A., Kruggel, F., and Von Cramon, D. Y. 1999. Segmentation of white matter lesions from volumetric MR images. 
In Lecture Notes in Computer Science(C. Taylor and A. Colchester, Eds.), Vol. 1679, pp. 52-61. Springer.

Hulshoff Pol, H. E., Hoek, H. W., Susser, E., Brown, A. S., Dingemans, A., Schnack, H. G., Van Haren, N. E. M., Ramos, L. M. P., Gispen-de Wied, C. C., and Kahn, R. S. 2000a. Prenatal exposure to famine and brain morphology in schizophrenia. Am. J . Psychiatry 157: 1170-1172.

Hulshoff Pol, H. E., Schnack, H. G., Bertens, M., Van Haren, N. E. M., Van der Tweel, I., Staal, W. G., Baaré, W. F. C., and Kahn, R. S. 2000b. Gray matter volume changes in schizophrenia. Submitted.

Kitagaki, H., Mori, E., Ishii, K., Yamaji, S., Hirono, N., and Imamura, T. 1998. CSF spaces inidiopathic normal pressure hydrocephalus: Morphology and volumetry. Am. J . Neuroradiol. 19: 1277-1284.

Kwan, R. K.-S., Evans, A. C., and Pike, G. B. 1996. An extensible MRI simulator for post-processing evaluation. Visualization in Biomedical Computing (VBC'96). Lecture N otes Comput. Sci. 1131: 135- 140. Springer Verlag.

Lawrie, S. M., and Abukmeil, S. S. 1998. Brain abnormality in schizophrenia. Br. J . Psychiatry 172: 110-120.

Leonard, C. M., Kuldau, J . M., Breier, J . I., Zuffante, P. A., Gautier, E. R., Heron, D.-C., Lavery, E. M., Packing, J ., Williams, S. A., and DeBose, C. A. 1999. Cumulative effect of anatomical risk factors for schizophrenia: An MRI study. Biol. Psychiatry 46: 374-382.

Maes, F., Collignon, A., Vandermeulen, D., Marchal, G., and Suetens, P. 1997. Multi-modality image registration by maximizing of mutual information. IEEE Trans. Med. Imag. 16: 187-198.

McCarley, R. W., Wible, C. G., Frumin, M., Hirayasu, Y., Levitt, J . L., Fischer, I. A., and Shenton, M. E. 1999. MRI Anatomy of Schizophrenia. Biol. Psychiatry 45: 1099-1119.

Mu, Q., Xie, J., Wen, Z., Weng, Y., and Shuyun, Z. 1999. A quantitative MR study of the hippocampal formation, the amygdala, and the temporal horn of the lateral ventricle in healthy subjects 40 to 90 years of age. Am. J. Neuroradiol. 20: 207-211.

Puri, B. K., Richardson, A. J ., Oatridge, A., Hajnal, J . V., and Saeed, N. 1999. Cerebral ventricular asymmetry in schizophrenia: A high resolution 3-D magnetic resonance imaging study. Int. J . Psychophysiol. 34: 207-211.

Rossi, A., Bustini, M., Prosperini, P., Marinangeli, M. G., Splendiani, A., Daneluzzo, E., and Stratta, P. 2000. Neuromorphological abnormalities in schizophrenic patients with good and poor outcome. Acta Psychiatr. Scand. 101: 161-166.

Roy, P. D., Zipursky, R. B., Saint-Cyr, J . A., Bury, A., Langevin, R., and Seeman, M. V. 1998. Temporal horn enlargement is present in schizophrenia and bipolar disorder. Biol. Psychiatry 44: 418- 422.
Saeed, N., Puri, B. K., Oatridge, A., Hajnal, J . V., and Young, I. R. 1998. Two methods for semi-automated quantification of changes in ventricular volume and their use in schizophrenia. Magn. Res. Imaging 16: 1237-1247.

Schnack, H. G., Hulshoff Pol, H. E., Baaré, W. F. C., Staal, W. G., Viergever, M. A., and Kahn, R. S. 2001. Automated separation of gray and white matter from MR images of the human brain. Neurol mage 13: 230-237.

Serra, J . 1982. Image Analysis and Mathematical Morphology. Academic Press, London.

Sled, J . G., Zijdenbos, A. P., and Evans, A. C. 1998. A nonparametric method for automatic correction of intensity nonuniformity in MRI data. IEEE Trans. Med. Imag. 17: 87-97.

Soares, J . C., and Mann, J . J . 1997. The anatomy of mood disordersReview of structural neuroimaging studies. Biol. Psychiatry $\mathbf{4 1}$ 86-106.

Staal, W. G., Hulshoff Pol, H. E., Schnack, H. G., Hoogendoorn, M. L. C., J ellema, K., and Kahn, R. S. 2000. Structural brain abnormalities in patients with schizophrenia and their healthy siblings. Am. J . Psychiatry 157: 416- 421.

Talairach, J ., and Tournoux, P. 1988. Co-planar Stereotaxic Atlas of the Human Brain. 3-Dimensional Proportional System: An Approach to Cerebral Imaging. Thieme, New York.

Visser, P. J ., Krabbendam, L., Verhey, F. R. J ., Hofman, P. A. M., Verhoeven, W. M. A., Tuinier, S., Wester, A., Van den Berg, Y. W. M. M., Goessens, L. F. M., Van der Werf, Y. D., and J olles, J . 1999. Brain correlates of memory dysfunction in alcoholic Korsakoff's syndrome. J . Neurol. Neurosurg. Psychiatry 67: 774-778.

Woodruff, P. W. R., Wright, I. C., Shuriquie, N., Russouw, H., Rushe, T., Howard, R. J ., Graves, M., Bullmore, E. T., and Murray, R. M. 1997. Structural brain abnormalities in male schizophrenics reflect fronto-temporal dissociation. Psychol. Med. 27: 1257-1266.

Worth, A. J ., Makris, N., Patti, M. R., Goodman, J . M., Hoge, E. A., Caviness, V. S., and Kennedy, D. N. 1998. Precise segmentation of the lateral ventricles and caudate nucleus in MR brain images using automatically driven histograms. IEEE Trans. Med. I maging 17: 303-310.

Wright, I. C., Rabe-Hesketh, S., Woodruff, P. W. R., David, A. S., Murry, R. M., and Bullmore, E. T. 2000. Meta-analysis of regional brain volumes in schizophrenia. Am. J . Psychiatry 157: 16-25.

Zijdenbos, A. P., Dawant, B. M., Margolin, R. A., and Palmer, A. C. 1994. Morphometric analysis of white matter lesions in MR images: Method and validation. IEEE Trans. Med. Imag. 13: 716724. 